\title{
REDUÇÃO DAS CORRENTES DE ARCO SECUNDÁRIO EM SISTEMAS DE TRANSMISSÃO TÍPICOS
}

\author{
Milton E. Zevallos* \\ zevallos@dsce.fee.unicamp.br
}

\author{
Maria C. Tavares* \\ cristinaddsce. fee. unicamp.br
}

${ }^{*}$ DSCE, FEEC, UNICAMP.

Caixa Postal 6101

CEP 13083-970 - Campinas SP

\section{RESUMO}

Neste trabalho otimizamos o valor de reator de neutro para conseguir minimizar a corrente de arco secundário em sistemas de transmissão com compensação reativa em derivação. Através de uma análise de sensibilidade conseguimos identificar valores de reator de neutro para os quais se consegue uma alta probabilidade de auto-extinção da corrente de arco secundário para diferentes sistemas de transmissão visando assegurar uma manobra abertura/religamento monopolar com sucesso. O método para identificar o reator de neutro ótimo e os valores das correntes de arco secundário para linhas típicas do sistema brasileiro é apresentado.

PALAVRAS-CHAVE: Arco Secundário, Compensação Reativa, Manobra Monofásica, Quadripolos e Reator de Neutro.

\section{ABSTRACT}

In this work we optimize the neutral reactor value in order to minimize the secondary arc current in transmission systems with shunt reactive compensation. Sensitivity analysis was conducted to identify neutral reactor values that will promote high probability of self-extinction the secondary arc current in different transmission systems to ensure a success singlephase opening/reclosure maneuver. The method is presented to identify the optimum neutral reactor and also values of the secondary arc current for typical lines of Brazilian system.

Artigo submetido em 22/07/2008 (Id.: 00883)

Revisado em 10/12/2008, 03/03/2009, 17/03/2009

Aceito sob recomendação do Editor Associado Prof. Eduardo N. Asada
KEYWORDS: Secondary Arc, Reactive Compensation, Single-phase Maneuver, Two-ports Systems and Neutral Reactor.

\section{INTRODUÇÃO}

Estatisticamente os defeitos monofásicos para terra com características não permanentes são os defeitos de maior ocorrência em um sistema de transmissão. A confiabilidade de um tronco de transmissão de energia aumenta se os curtoscircuitos não permanentes forem eliminados através da manobra de abertura monofásica e um posterior religamento rápido da fase sob falta, sem que se interrompa o fornecimento de energia para os centros de carga.

Uma abertura trifásica da linha, com um posterior religamento trifásico rápido pode acarretar alguns efeitos negativos na estabilidade e confiabilidade do sistema elétrico (Gonzales et alii, 1984; EPRI, 1982) com risco de propagação do defeito (efeito tipo "cascata"). Uma abertura monofásica reduz esse tipo de risco, podendo continuar a transmitir grandes blocos de potência através das fases sem distúrbio (Kimbark,1976), que não são desligadas.

Após a abertura de ambos os extremos da fase com defeito no caso de faltas monofásicas para terra, a corrente de falta é reduzida para uma condição comumente denominada "corrente de arco secundário" (Isec). Antes que o religamento monofásico seja executado, é necessário que o arco secundário seja extinto e que a tensão pós arco seja pequena.

Após a extinção do arco, o ar na região do defeito continua 
ionizado. Caso a tensão no ponto do defeito, aqui denominada tensão pós-arco (TPA), seja muito elevada pode ocorrer a reignição do arco. Desta forma para que haja uma alta probabilidade de o religamento monofásico ter sucesso é necessário que a corrente de arco secundário se extinga e que a TPA seja pequena.

Nos sistemas de transmissão é adequado otimizar os parâmetros que possam contribuir para um melhor desempenho e confiabilidade do transporte de energia. Em nossa análise reduzimos a corrente de arco secundário através da otimização do reator de neutro em sistemas com bancos de reatores em derivação. A análise foi feita para linhas típicas do sistema brasileiro estudando o caso de energização da linha sob falta monofásica.

\section{OTIMIZAÇÃO DO REATOR DE NEUTRO}

A corrente de arco secundário é mantida principalmente pelo acoplamento capacitivo entre as fases sãs e a fase interrompida no caso de faltas monofásicas para terra de caráter não permanente sendo um fenômeno basicamente a freqüência fundamental.

Nas linhas longas de transmissão se utiliza a compensação reativa em derivação (configuração "quatro pernas") (Kimbark,1964) para o controle da tensão em regime permanente, assim como para a redução das sobretensões de manobra. A otimização deste equipamento é o método utilizado para reduzir e até eliminar o acoplamento capacitivo entre as fases. Em nosso trabalho a hipótese básica assumida consiste em supor que o arco secundário é composto basicamente pela frequiência fundamental de modo que se procurou o reator de neutro que minimizasse o acoplamento capacitivo nesta freqüência. Limitamos a corrente e tensão pós-arco dimensionando adequadamente os valores do reator de neutro (Tavares e Portela, 2004) para um nível de compensação reativa definido para o sistema.

Neste artigo não se utiliza a modelagem de arco em função do tempo nem procedimentos de interação arco-rede (Portela et alii, 1992; Portela, 1980). Da mesma forma a metodologia proposta não se aplica a troncos de transmissão muito longos, com comprimentos superiores a $1 / 4$ do comprimento de onda.

Troncos em corrente alternada (CA) com comprimentos muito longos, por exemplo, acima de $2000 \mathrm{~km}$, devem ter seu sistema de compensação reativo otimizado de forma diferente dos troncos usuais de poucas centenas de quilômetros. Os troncos usuais são encurtados eletricamente através da instalação de bancos de reatores em derivação e muitas vezes de capacitores série. No caso de troncos muito longos pode ser mais conveniente alongar eletricamente a linha, fazendo com que ele opere como um tronco com um pouco mais de $1 / 2$ comprimento de onda. Neste caso, se necessário, a compensação utilizada será diferente da convencional, especificamente será composta por bancos de capacitores em derivação e reator série.

A forma mais simples e de menor custo para se reduzir a corrente de arco secundário em linhas não muito longas é otimizar o reator de neutro para se minimizar o acoplamento capacitivo entre as fases. Como para troncos muito longos não se utiliza reator em derivação, não existe reator de neutro para ser otimizado. Outras formas de redução da corrente de arco secundário para estes troncos estão sendo desenvolvidas e serão apresentadas em trabalhos futuros.

\section{REPRESENTAÇÃO TRIFILAR DOS PRINCIPAIS ELEMENTOS DOS SISTE- MAS EM ESTUDO}

Para se efetuar a análise de sensibilidade a freqüência fundamental todos os elementos do sistema foram representados no domínio da frequiência. Para tal foi desenvolvido um programa que permitisse a representação trifásica dos componentes do sistema, tendo-se trabalhado com quadripolos trifásicos. Nesta seção os elementos principais utilizados são descritos (Zevallos, 2007).

\subsection{Curto Circuito Monofásico para Terra}

Um curto circuito monofásico para terra é representado por uma impedância de valor baixo. Como o objetivo do trabalho é obter o valor da Isec, foi utilizado um baixo valor de impedância de falta, especificamente $\left(Z_{f}=1 \Omega\right)$. Para baixos valores de impedância de falta, a amplitude da parcela sustentada $(60 \mathrm{~Hz})$ da corrente de arco secundário não deverá variar muito, pois esta é função da tensão em vazio no ponto de defeito e da impedância equivalente do sistema vista do ponto de falta, com os terminais abertos da fase sob falta. Esta impedância equivalente varia para cada sistema, mas se, por hipótese, for da ordem de $1000 \Omega$, a corrente de arco obtida não irá variar para pequenos valores de impedância de falta. Valores elevados de impedância de falta, da ordem de $500 \Omega$, podem alterar o valor de Isec neste exemplo. Deve-se ter cautela ao se utilizar valores elevados de impedância de falta sob o risco de se obter correntes excessivamente baixas. $\mathrm{Na}$ figura 1 mostramos o esquema respectivo de uma falta monofásica para terra na fase "a". Em (1) e (2) resume-se as tensões e correntes na fase com defeito.

$$
\begin{gathered}
U_{2 a}=U_{1 a} \\
I_{2 a}=I_{1 a}-\frac{U_{1 a}}{Z_{f}}
\end{gathered}
$$




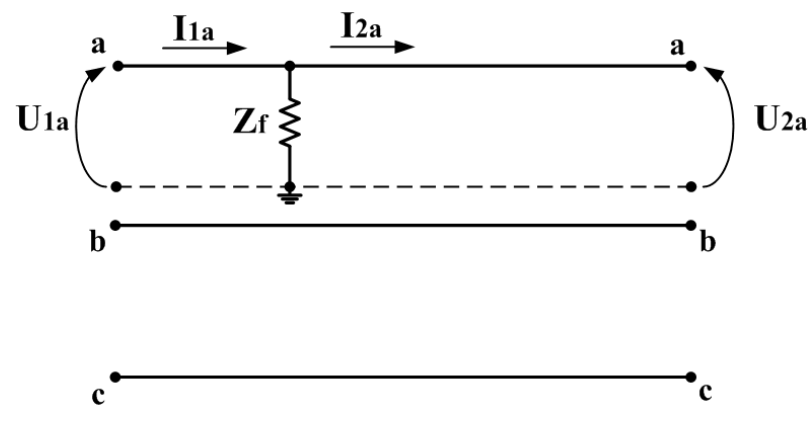

Figura 1: Diagrama Trifilar de um Curto Circuito Monofásico para Terra na Fase "a".

Onde $\mathrm{U}_{1 a}, \mathrm{U}_{2 a}, \mathrm{I}_{1 a}$ e $\mathrm{I}_{2 a}$ são as tensões e correntes nas portas do circuito na fase "a". A expressão matricial monofásica é como segue:

$$
\left[\begin{array}{c}
U_{2 a} \\
I_{2 a}
\end{array}\right]=\left[\begin{array}{cc}
1 & 0 \\
\frac{-1}{Z_{f}} & 1
\end{array}\right] \cdot\left[\begin{array}{c}
U_{1 a} \\
I_{1 a}
\end{array}\right]
$$

Onde $\mathrm{Z}_{f}$ equivale a uma baixa impedância que conduz a corrente de curto-circuito que queremos representar.

\subsection{Compensação Reativa em Derivação}

A compensação em derivação para linhas com comprimento longo permite o controle da tensão em regime permanente. Aproveitando a configuração "quatro pernas" podemos minimizar o acoplamento capacitivo entre as fases sãs e a fase com falta através do correto dimensionamento do reator de neutro. O quadripolo do banco de reator trifásico com neutro é mais facilmente entendido analisando-se inicialmente a sua representação no domínio dos modos.

Na figura 2 mostra-se o equivalente trifásico da compensação em derivação. $\mathrm{X}_{f}$ é o reator de fase do banco de compensação em derivação e $\mathrm{X}_{n}$ é o reator de neutro.

$$
\begin{aligned}
& X_{d}=X_{f} \\
& Y_{d}=\frac{1}{X_{d}}
\end{aligned}
$$

A reatância de fase $\left(\mathrm{X}_{f}\right)$ é igual à reatância não homopolar $\left(\mathrm{X}_{d}\right)$ da compensação transversal da linha. $\mathrm{Y}_{d}$ é a correspondente admitância não homopolar. A reatância homopolar $\left(\mathrm{X}_{0}\right)$ da compensação transversal é obtida através de (6).

$$
X_{0}=X_{f}+3 \cdot X_{n}
$$

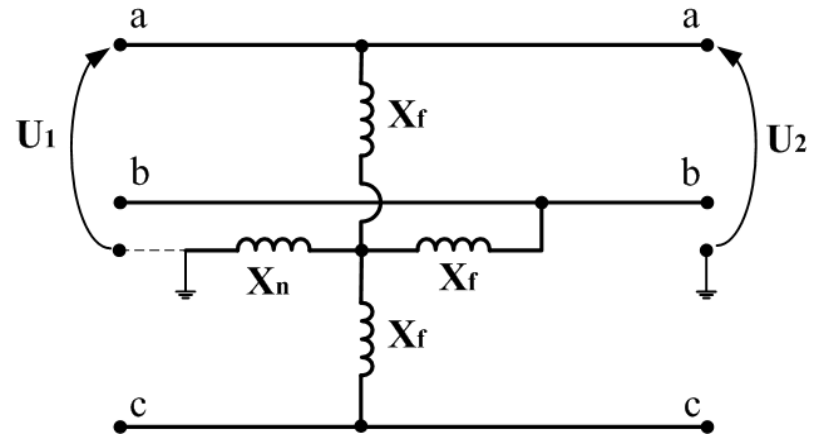

Figura 2: Diagrama Trifásico da Compensação Transversal.

$$
\begin{gathered}
r_{0}=\frac{X_{0}}{X_{d}}=\frac{X_{f}+3 \cdot X_{n}}{X_{f}} \\
r_{0}=\frac{\frac{1}{Y_{0}}}{\frac{1}{Y_{d}}}=\frac{Y_{d}}{Y_{0}}
\end{gathered}
$$

$\mathrm{Y}_{0}$ é a admitância homopolar correspondente. Para nossa análise utilizamos a relação " $\mathrm{r}_{0}$ " para efetuar a análise de sensibilidade. A expressão matricial em modos de seqüência positiva é como segue:

$$
\left[\begin{array}{c}
U_{2 d} \\
I_{2 d}
\end{array}\right]=\left[\begin{array}{cc}
1 & 0 \\
-1 X_{d} & 1
\end{array}\right] \cdot\left[\begin{array}{c}
U_{1 d} \\
I_{1 d}
\end{array}\right]
$$

A representação modal completa dos reatores através das admitâncias é descrita em (10). Este quadripolo em modos foi transformado para quadripolo trifásico em cada ponto de compensação transversal da linha utilizando a transformada de Clarke (11a) e a transformada inversa de Clarke (11b). Em (11) mostra-se como foi essa transformação.

$$
\begin{gathered}
Q_{R m}=\left[\begin{array}{cccccc}
1 & 0 & 0 & 0 & 0 & 0 \\
0 & 1 & 0 & 0 & 0 & 0 \\
0 & 0 & 1 & 0 & 0 & 0 \\
-Y_{0} & 0 & 0 & 1 & 0 & 0 \\
0 & -Y_{d} & 0 & 0 & 1 & 0 \\
0 & 0 & -Y_{d} & 0 & 0 & 1
\end{array}\right] ; \\
Q_{R f}=T c l^{-1} \cdot Q_{R m} \cdot T c l \\
T_{C l}^{-1}=\left[\begin{array}{ccc}
2 / \sqrt{6} & -1 / \sqrt{6} & -1 / \sqrt{6} \\
0 & 1 / \sqrt{2} & -1 / \sqrt{2} \\
1 / \sqrt{3} & 1 / \sqrt{3} & 1 / \sqrt{3} \\
2 / \sqrt{6} & 0 & 1 / \sqrt{3} \\
-1 / \sqrt{6} & 1 / \sqrt{2} & 1 / \sqrt{3} \\
-1 / \sqrt{6} & -1 / \sqrt{2} & 1 / \sqrt{3}
\end{array}\right]
\end{gathered}
$$




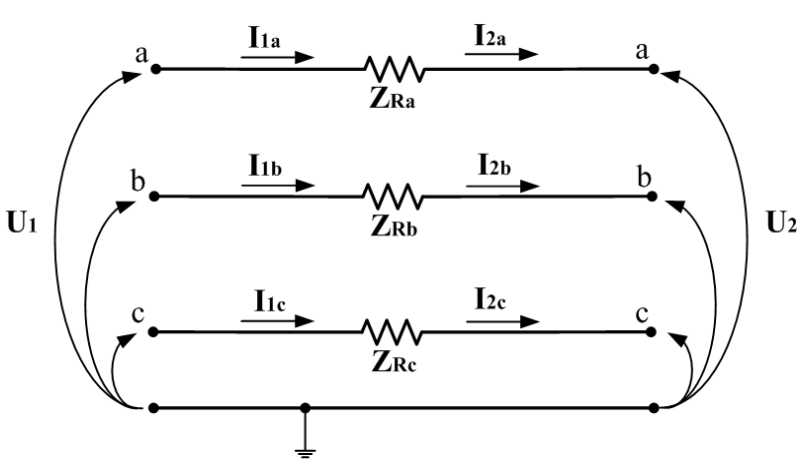

Figura 3: Diagrama Trifilar da Representação do Disjuntor em cada Fase.

\subsection{Disjuntores}

Um disjuntor fechado ou aberto é representado através de uma resistência nula $\left(Z_{R}=0\right)$ ou de valor elevado $\left(\mathrm{Z}_{R}=10^{6} \Omega\right.$ ), respectivamente. A figura 3 representa o esquema deste dispositivo com a notação para cada representação do disjuntor de fase.

Onde $\mathrm{Z}_{R a}$ é a representação do disjuntor da fase "a", $\mathrm{Z}_{R b}$ é a representação do disjuntor da fase "b" e $Z_{R c}$ a representação do disjuntor da fase "c".

$$
\begin{gathered}
U_{2}=U_{1}-Z_{R} \cdot I_{R} \\
I_{2}=I_{R}
\end{gathered}
$$

Onde $\mathrm{U}_{1}, \mathrm{U}_{2}, \mathrm{I}_{R}$ e $\mathrm{I}_{2}$ são as tensões e correntes nas portas do circuito monofásico da figura 3. A expressão matricial monofásica é como segue:

$$
\left[\begin{array}{c}
U_{2} \\
I_{2}
\end{array}\right]=\left[\begin{array}{cc}
1 & -Z_{R} \\
0 & 1
\end{array}\right] \cdot\left[\begin{array}{c}
U_{1} \\
I_{1}
\end{array}\right]
$$

Onde $Z_{R}$ equivale a uma baixa ou uma alta impedância para que o disjuntor representado possa respectivamente conduzir (disjuntor fechado) ou impedir (disjuntor aberto) a passagem da corrente elétrica.

\section{SISTEMAS DE TRANSMISSÃO ANALI- SADOS}

Neste trabalho analisamos sistemas de $345 \mathrm{kV}, 500 \mathrm{kV}$ e $765 \mathrm{kV}$, com torres típicas em disposição convencional de seus condutores. Analisamos também uma linha de $500 \mathrm{kV}$ com disposição não convencional dos seus condutores. Previamente calculamos os parâmetros elétricos das linhas em estudo através de um programa desenvolvido em ambiente

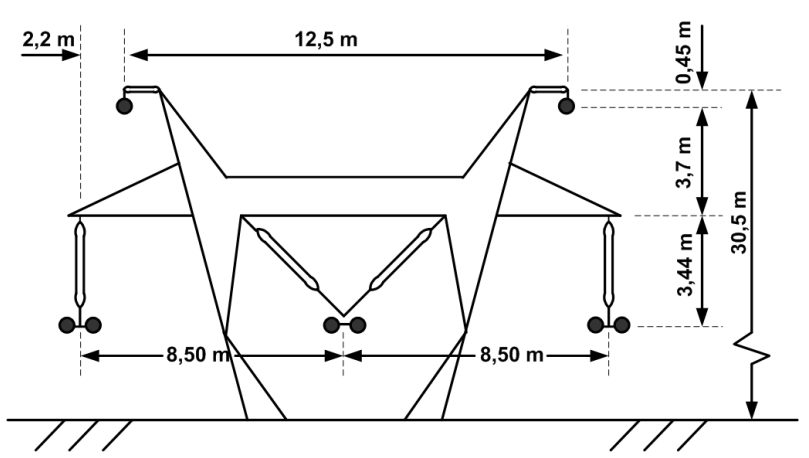

Figura 4: Silhueta da Torre da Linha Convencional de 345 $\mathrm{kV}$ em Estudo.

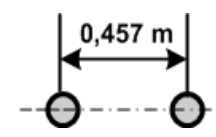

Figura 5: Detalhe do Feixe na Linha Convencional de 345 $\mathrm{kV}$ em Estudo.

Matlab @ 7.0.1. Estes valores podem ser obtidos em programas do tipo ATP (Alternative Transient Program). Nas tabelas 1, 2 e 3 mostram-se os parâmetros elétricos obtidos para cada sistema analisado, também é mostrada a potência característica de cada linha em estudo.

Na figura 4 mostra-se a silhueta superior da torre de $345 \mathrm{kV}$ estudada com as distâncias entre condutores e entre fases,

\begin{tabular}{|c|c|c|}
\hline \multicolumn{3}{|c|}{ Linha Convencional } \\
\hline \multirow{3}{*}{ 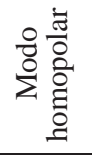 } & $\mathbf{R}_{0 k m}(\Omega / \mathbf{k m})$ & 0,3487 \\
\hline & $\mathbf{X}_{0 k m}(\Omega / \mathbf{k m})$ & 1,5495 \\
\hline & $\mathbf{Y}_{0 k m}(\mu \mathbf{S} / \mathbf{k m})$ & 3,354 \\
\hline \multirow{3}{*}{ 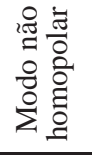 } & $\mathbf{R}_{1 k m}(\Omega / \mathbf{k m})$ & 0,0306 \\
\hline & $\mathbf{X}_{1 k m}(\Omega / \mathbf{k m})$ & $\mathbf{0 , 3 7 5 3}$ \\
\hline & $\mathbf{Y}_{1 k m}(\mu \mathbf{S} / \mathbf{k m})$ & 4,517 \\
\hline \multicolumn{2}{|c|}{$\begin{array}{c}\text { Potência Característica } \\
\text { (MW) }\end{array}$} & 412,25 \\
\hline
\end{tabular}
assim como suas alturas na torre.

Tabela 1: Parâmetros Elétricos da Linha de 345 kV a 60 Hz.

O cabo condutor usado para esta linha de $345 \mathrm{kV}$ foi do tipo Rail. A flecha no meio do vão entre duas torres foi $14,6 \mathrm{~m}$. $\mathrm{Na}$ figura 5 mostra-se o detalhe do feixe neste sistema.

O cabo pára-raios é um cabo sólido do tipo EHS 3/8", a flecha no meio do vão entre duas torres é $10,62 \mathrm{~m}$.

Tabela 2: Parâmetros Elétricos da Linha de 500 kV a 60 Hz. 
Tabela 2: Parâmetros Elétricos da Linha de 500 kV a 60 Hz.

\begin{tabular}{|c|c|c|c|}
\hline \multicolumn{2}{|c|}{ Tipo de Linha } & Convencional & $\begin{array}{l}\text { Não Con- } \\
\text { vencional }\end{array}$ \\
\hline \multirow{3}{*}{ 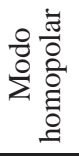 } & $\mathbf{R}_{0 k m}(\Omega / \mathbf{k m})$ & 0,3235 & 0,3235 \\
\hline & $\mathbf{X}_{0 k m}(\Omega / \mathbf{k m})$ & 1,5504 & 1,3448 \\
\hline & $\mathbf{Y}_{0 k m}(\mu \mathbf{S} / \mathbf{k m})$ & 2,729 & 3,79 \\
\hline \multirow{3}{*}{ 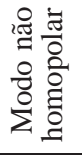 } & $\mathbf{R}_{1 k m}(\Omega / \mathbf{k m})$ & 0,0154 & 0,0155 \\
\hline & $\mathbf{X}_{1 k m}(\Omega / \mathbf{k m})$ & 0,2670 & 0,2294 \\
\hline & $\mathbf{Y}_{1 k m}(\mu \mathbf{S} / \mathbf{k m})$ & 6,18 & 7,283 \\
\hline \multicolumn{2}{|c|}{$\begin{array}{c}\text { Potência } \\
\text { Característica (MW) }\end{array}$} & 1199,83 & 1407,03 \\
\hline
\end{tabular}

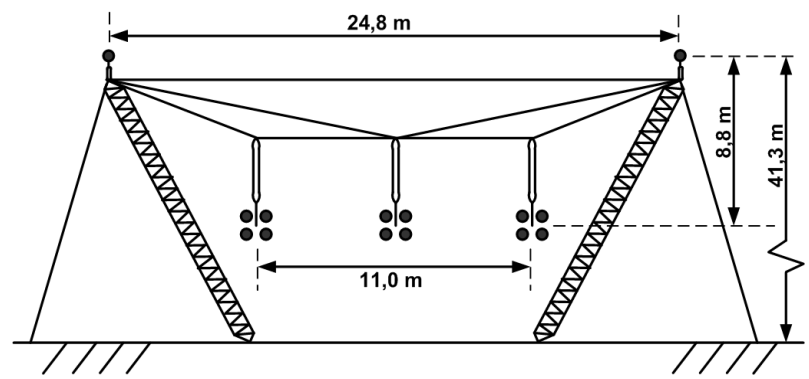

Figura 6: Silhueta da Torre da Linha Convencional de 500 $\mathrm{kV}$ em Estudo.

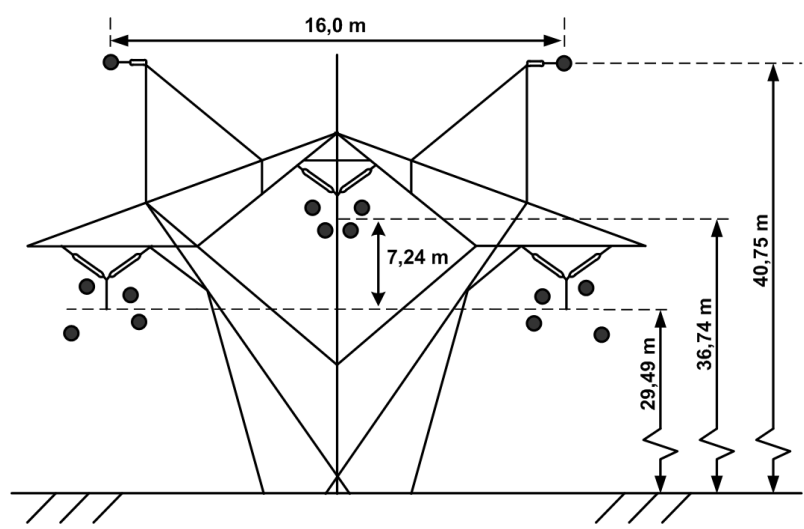

Figura 7: Silhueta da Torre da Linha não Convencional de $500 \mathrm{kV}$ em Estudo.

Nas figura 6 e 7 mostram-se a silhueta superior de uma torre típica em $500 \mathrm{kV}$ de características convencionais e a silhueta superior de uma torre não convencional de $500 \mathrm{kV}$ respectivamente, assim como as distâncias entre condutores e entre fases, e suas alturas na torre.

O cabo condutor usado para a linha convencional e não convencional de $500 \mathrm{kV}$ foi do tipo Rail. A flecha no meio do

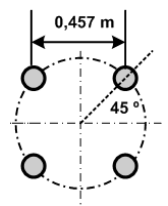

A)

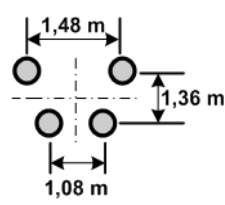

B)

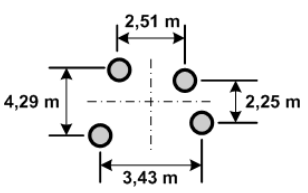

C)
Figura 8: A) Detalhe do Feixe na Linha Convencional de 500 kV em Estudo, B) Detalhe do Feixe da Fase Central na Linha não Convencional de $500 \mathrm{kV}$ em Estudo e,C) Detalhe dos Feixes das Fases Externas na Linha não Convencional de $500 \mathrm{kV}$ em Estudo.

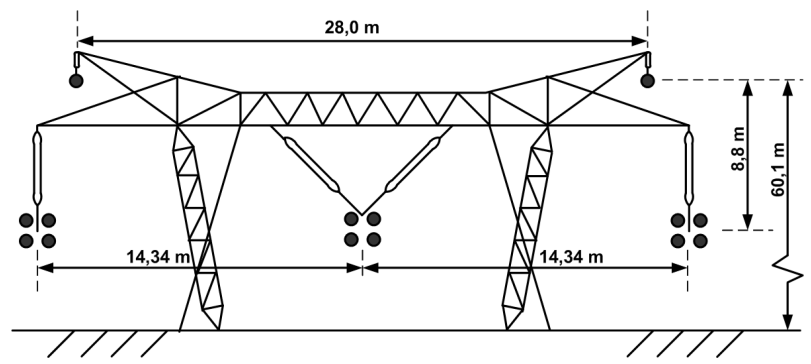

Figura 9: Silhueta da Torre da Linha Convencional de 765 $\mathrm{kV}$ em Estudo.

vão entre duas torres de ambos sistemas foi $13,43 \mathrm{~m}$. Na figura 8A mostra-se o detalhe do feixe no sistema convencional, nas figuras $8 \mathrm{~B}$ e $8 \mathrm{C}$ mostram-se os detalhes do feixe central e do feixe das fases externas no sistema não convencional.

O cabo pára-raios para os sistemas convencional e não convencional de $500 \mathrm{kV}$ é um cabo sólido do tipo EHS 3/8", a flecha no meio do vão entre duas torres é $6,4 \mathrm{~m}$.

Tabela 3: Parâmetros Elétricos da Linha de 765 kV a 60 Hz.

\begin{tabular}{|c|c|c|}
\hline \multicolumn{3}{|c|}{ Linha Convencional } \\
\hline \multirow{3}{*}{ 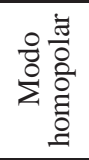 } & $\mathbf{R}_{0 k m}(\Omega / \mathbf{k m})$ & 0,3478 \\
\hline & $\mathbf{X}_{0 k m}(\Omega / \mathbf{k m})$ & 1,4015 \\
\hline & $\mathbf{Y}_{0 k m}(\mu \mathbf{S} / \mathbf{k m})$ & 3,404 \\
\hline \multirow{3}{*}{ 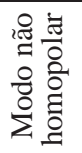 } & $\mathbf{R}_{1 k m}(\Omega / \mathbf{k m})$ & 0,0165 \\
\hline & $\mathbf{X}_{1 k m}(\Omega / \mathbf{k m})$ & 0,3651 \\
\hline & $\mathbf{Y}_{1 k m}(\mu \mathbf{S} / \mathbf{k m})$ & 4,759 \\
\hline \multicolumn{2}{|c|}{ Potência Característica (MW) } & 2111,81 \\
\hline
\end{tabular}

Na figura 9 mostra-se a silhueta superior de uma torre em $765 \mathrm{kV}$ com as distâncias entre condutores e entre fases, assim como suas alturas na torre.

O cabo condutor usado para esta linha de $765 \mathrm{kV}$ foi do tipo 
Bluejay. A flecha no meio do vão entre duas torres foi 13,43 $\mathrm{m}$. O detalhe do feixe neste sistema é igual ao sistema convencional de $500 \mathrm{kV}$ (Ver figura 8A).

O cabo pára-raios é um cabo sólido do tipo EHS 3/8", a flecha no meio do vão entre duas torres é $6,4 \mathrm{~m}$.

\section{ANÁLISE DE RESULTADOS}

A eliminação do defeito monofásico não permanente através de uma manobra monofásica tem elevada probabilidade de sucesso se o valor eficaz da corrente de arco secundário for reduzido. Nesta análise trabalhamos por hipótese com os seguintes limites:

- Corrente de arco secundário, Isec menor ou igual a $50 \mathrm{~A}$ - arcos com alta probabilidade de se auto-extinguirem.

- Corrente de arco secundário maior do que 100 A - arcos com baixa probabilidade de se auto-extinguirem.

- Arcos secundários com valor eficaz entre 50 e 100 A encontram-se em uma região difusa onde é necessário efetuar uma representação mais precisa do arco e do sistema elétrico.

Deve-se ressaltar que esta análise a regime permanente é adequada para se identificar o nível de arco secundário sustentado e se otimizar o sistema de modo a minimizar este valor. A partir da obtenção da corrente de arco secundário máximo que pode ocorrer para o sistema de transmissão otimizado é necessário analisar o comportamento da interação do arco com a rede no domínio do tempo para se verificar se o arco se torna instável e, portanto, tem alta probabilidade de se extinguir. Para isto é preciso representar o arco através de um modelo que represente corretamente sua resposta dinâmica junto com a rede elétrica.

As faixas de corrente de arco secundário para as quais haveria alta ou baixa probabilidade de extinção de arco foram arbitradas. Para se verificar se a manobra monopolar num sistema, por exemplo, com Isec de $100 \mathrm{~A}_{e f}$ terá sucesso, é necessário efetuar análises no domínio do tempo. É possível que arcos desta magnitude se extingam, mas isto somente poderá ser definido com simulações no domínio do tempo. Por outro lado, arcos com correntes sustentadas muito baixas, de poucas dezenas de amperes têm alta probabilidade de extinção, mas sempre é preferível efetuar verificações no domínio do tempo.

Apesar de no trabalho ter sido apresentado o valor da tensão pós-arco no ponto de defeito, este valor não foi utilizado para avaliar a probabilidade de sucesso da manobra.
Efetuamos assim a análise de sensibilidade para as linhas em estudo com diferentes níveis de tensão, comprimentos de linha e graus de compensação reativa. Identificamos a maior corrente eficaz de arco secundário que pode ocorrer otimizando o reator de neutro uma vez que o reator de fase é definido pelo nível de compensação desejado. Então, para este propósito desenvolvemos um programa em ambiente Matlab @ $7.01 \mathrm{com}$ a finalidade de obter ótimos valores de reator de neutro. Variamos o valor do reator de neutro através da variação da relação " $\mathrm{r}_{0}$ " em um intervalo de 1 até 6 , com o objetivo de encontrar o " $r_{0}$ " que gere a menor Isec máxima nos casos de defeitos monofásicos não permanentes para terra simulados a cada $20 \mathrm{~km}$ ao longo da linha durante a manobra de energização da linha. A rede a montante foi representada como uma barra infinita. Toda a análise foi realizada observando somente a resposta sustentada do sistema $(60 \mathrm{~Hz})$.

Levamos em consideração os limites de ganho de tensão no final da linha para os graus de compensação simulados.

A seguir, apresentamos os resultados para os diferentes sistemas começando com as linhas sem compensação, linhas com compensação reativa em derivação e linhas com compensação reativa em derivação e compensação capacitiva série.

\subsection{Análise de Resultados em Linhas sem Compensação}

Para linhas sem compensação consideramos dois comprimentos de linha, uma linha curta $(150 \mathrm{~km})$ e uma linha longa (450 km).

Na tabela 4 se mostram os resultados obtidos para estes casos, onde se pode afirmar que em linhas curtas sem compensação existe uma alta probabilidade que a Isec seja extinta para os casos de defeitos monofásicos em qualquer local ao longo da linha.

Ao contrário para a linha longa de $450 \mathrm{~km}$, os valores de Isec e TPA podem ser muito severos (Isec > $100 \mathrm{~A}$ ) em todas as classes de tensão simuladas. No entanto estes casos são somente ilustrativos, uma vez que estas linhas com $450 \mathrm{~km}$ precisariam de compensação reativa para reduzir o efeito Ferranti.

\subsection{Análise de Resultados em Linhas com Compensação Reativa em Deri- vação}

A figura 10 mostra o diagrama unifilar dos sistemas com compensação reativa em derivação, onde L é o comprimento total da linha e os bancos de reatores (R.Der) estão localizados nos extremos da linha. 
Tabela 4: Resultados para Linhas sem Compensação.

\begin{tabular}{|c|c|c|c|c|}
\hline \multirow{4}{*}{ Sistema } & $\mathbf{V}$ & $\mathbf{L}$ & $\mathbf{I s e c}$ & $\mathbf{T P A}$ \\
\cline { 2 - 5 } & $\mathbf{( k V )}$ & $\mathbf{( k m )}$ & $\mathbf{( A )}$ & $\mathbf{( k V )}$ \\
\hline \multirow{4}{*}{ Convencional } & 345 & 150 & $\mathbf{1 2 , 1}$ & 10,8 \\
\cline { 2 - 5 } & 345 & 450 & 110 & 38 \\
\cline { 2 - 5 } & 500 & 150 & $\mathbf{5 0 , 8}$ & 42,8 \\
\cline { 2 - 5 } & 500 & 450 & 405 & 104 \\
\cline { 2 - 5 } & 765 & 150 & $\mathbf{3 0 , 9}$ & 27,5 \\
\cline { 2 - 5 } & 765 & 450 & 220 & 98 \\
\hline \multirow{4}{*}{$\begin{array}{c}\text { Não } \\
\text { Convencional }\end{array}$} & 500 & 150 & $\mathbf{5 2 , 1}$ & 40,3 \\
\cline { 2 - 5 } & 500 & 450 & 475 & 100 \\
\hline
\end{tabular}

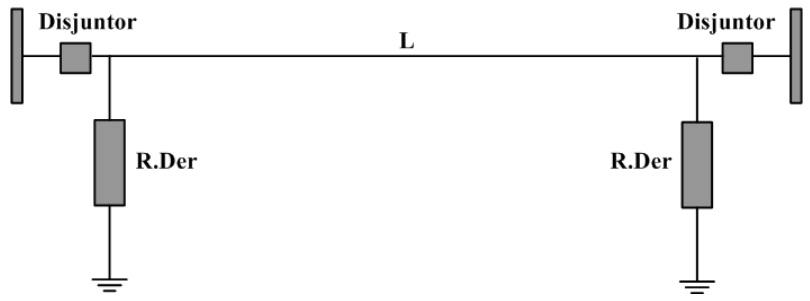

Figura 10: Diagrama Unifilar de uma Linha com Compensação Reativa em Derivação.

Para cada sistema de transmissão composto por linha e compensação reativa, o reator de neutro foi variado e para cada valor de reator de neutro foram obtidos os valores de Isec e TPA variando-se o local de aplicação de defeito. Os maiores valores de Isec e TPA para cada valor de reator de neutro são apresentados na figura 11 para a linha de $500 \mathrm{kV}$ convencional de $450 \mathrm{~km}$ com compensação reativa longitudinal de $70 \%$. A variação do reator de neutro é descrita através da variação de "ro". A razão " $\mathrm{r}_{0}$ " que corresponde ao menor valor de Isec para este sistema é 4,08. Na figura 12 apresentam-se os valores de Isec e TPA para faltas ao longo da linha para o " $\mathrm{r}_{0}$ " ótimo.

Na tabela 5 são mostrados os valores obtidos de Isec, TPA e reator de neutro $\left(\mathrm{X}_{n}\right)$ para cada nível de compensação em derivação simulado, para cada classe de tensão pré-estabelecida em linhas de $450 \mathrm{~km}$.

Pelos resultados obtidos pode-se afirmar que para os exemplos analisados o correto dimensionamento do reator de neutro foi suficiente para reduzir o valor da Isec para valores muito baixos, indicando uma alta probabilidade de autoextinção num tempo curto. O desejável é que a manobra de religamento possa ser efetuada entre 0,5 e $1 \mathrm{~s}$.

Tabela 5: Resultados para Linhas com Compensação em De-

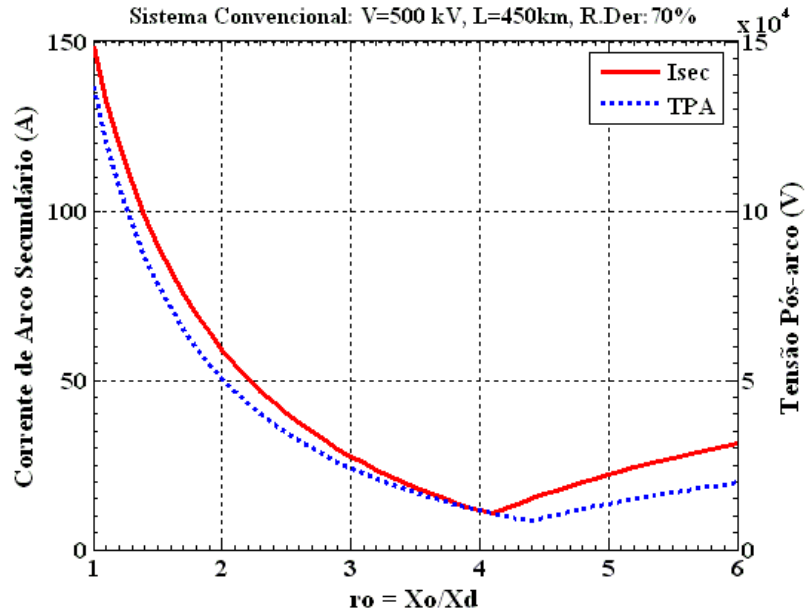

Figura 11: Valores Máximos de Isec e TPA em função de "ro" - Linha $500 \mathrm{kV}$ Convencional com Compensação de $70 \%(450 \mathrm{~km})$.

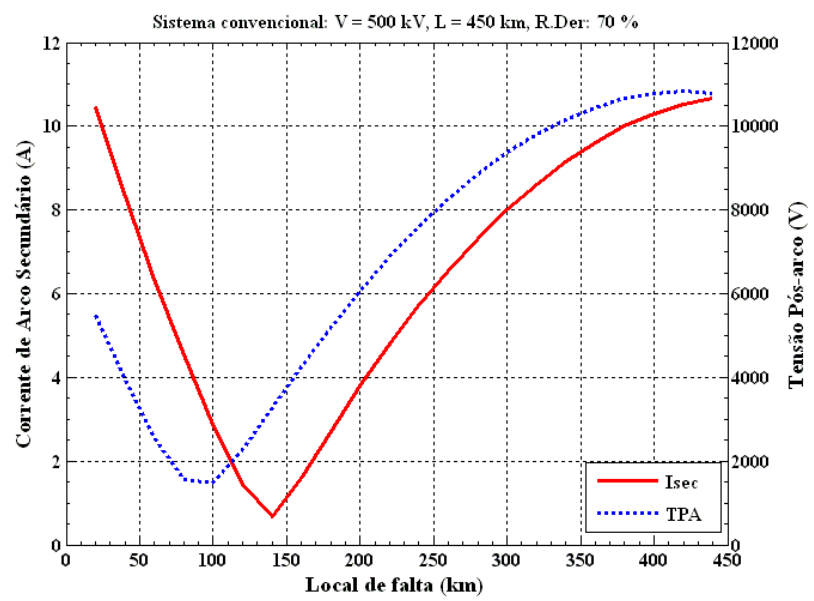

Figura 12: Isec e TPA ao Longo da Linha para o Reator de Neutro Ótimo $\left(\mathrm{r}_{0}=4,08\right)$ - Linha $500 \mathrm{kV}$ Convencional com Compensação de $70 \%$ (450 km).

rivação $(450 \mathrm{~km})$.

Na figura 13 mostra-se um exemplo de obtenção do valor de " $\mathrm{r}_{0}$ " ótimo $(1,9)$ para o sistema de $500 \mathrm{kV}$ convencional com comprimento $900 \mathrm{~km}$ e grau de compensação reativa $90 \%$. Este valor ótimo de " $\mathrm{r}_{0}$ " foi obtido encontrando a Isec máxima para faltas ao longo da linha ao variar-se " $\mathrm{r}_{0}$ ". $\mathrm{Na}$ figura 14 apresenta-se Isec e TPA para faltas ao longo da linha com "ro" ótimo.

Na tabela 6 são mostrados os valores obtidos de Isec, TPA e reator de neutro $\left(\mathrm{X}_{n}\right)$ para cada nível de compensação em derivação simulado, para cada classe de tensão pré-estabelecida em linhas de $900 \mathrm{~km}$. 
Tabela 5: Resultados para Linhas com Compensação em Derivação $(450 \mathrm{~km})$.

\begin{tabular}{|c|c|c|c|c|c|c|c|}
\hline \multirow{2}{*}{ Sistema } & V & $\mathbf{L}$ & R.Der & $\mathbf{r}_{0}$ & $\mathbf{X n}$ & Isec & TPA \\
\hline & $(\mathrm{kV})$ & $(\mathrm{km})$ & $(\%)$ & $\left(\frac{X_{0}}{X d}\right)$ & $(\Omega)$ & (A) & $(\mathrm{kV})$ \\
\hline \multirow{6}{*}{ 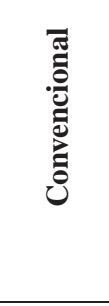 } & 500 & 450 & 70 & 4,1 & 1025,24 & 10,8 & 10,7 \\
\hline & 500 & 450 & 80 & 2,85 & 540,64 & 6 & 10,5 \\
\hline & 500 & 450 & 100 & 2,18 & 274,95 & 2 & 3 \\
\hline & 765 & 450 & 70 & 1,5 & 215,80 & 11,4 & 11,6 \\
\hline & 765 & 450 & 80 & 1,4 & 151,06 & 8,8 & 10,2 \\
\hline & 765 & 450 & 100 & 1,3 & 90,64 & 5,4 & 6,34 \\
\hline \multirow{3}{*}{ 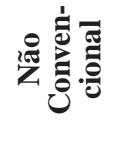 } & 500 & 450 & 70 & 2,7 & 465,89 & 13,6 & 11,6 \\
\hline & 500 & 450 & 80 & 2,23 & 303,88 & 6,5 & 8,8 \\
\hline & 500 & 450 & 100 & 1,8 & 148,24 & 4,3 & 3 \\
\hline
\end{tabular}

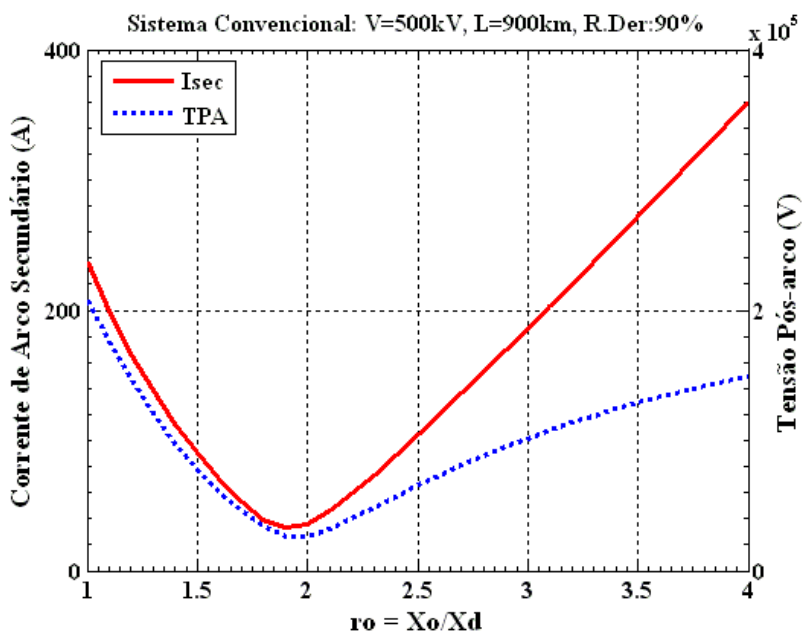

Figura 13: Valores Máximos de Isec e TPA em função de "ro" - Linha $500 \mathrm{kV}$ Convencional com Compensação de $90 \%(900 \mathrm{~km})$.

Para linhas muito longas, com comprimento um pouco inferior a $1 / 4$ do comprimento de onda, a otimização do reator de neutro não foi suficiente para garantir a auto-extinção do arco secundário com exceção da linha convencional de $500 \mathrm{kV}$. A compensação reativa destes casos foi concentrada somente nos extremos, o que não é adequado para linhas longas. $\mathrm{Na}$ próxima seção uma nova proposta de compensação mais distribuída ao longo da linha, reduzindo-se não somente a admitância transversal como também a indutância longitudinal será analisada.

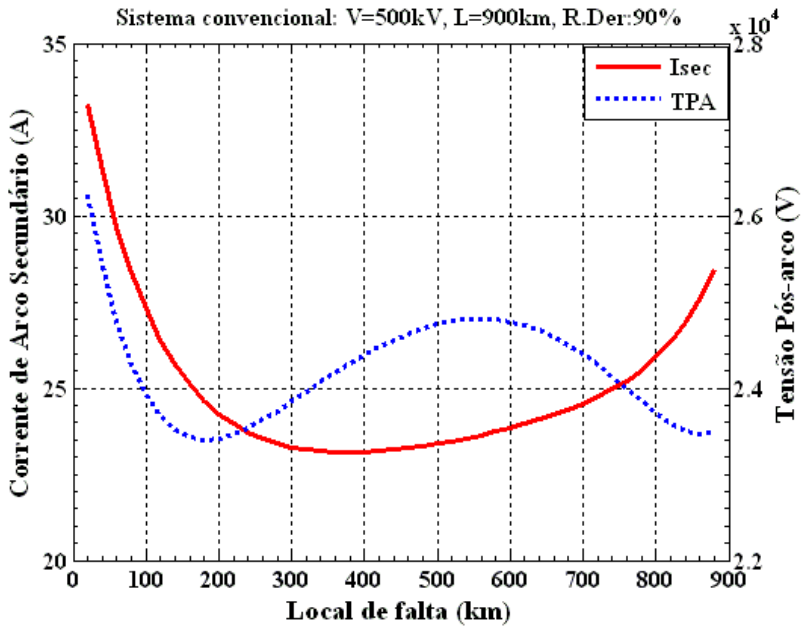

Figura 14: Isec e TPA ao Longo da Linha para o Reator de Neutro Ótimo $\left(\mathrm{r}_{0}=1,9\right)$ - Linha $500 \mathrm{kV}$ Convencional com Compensação de $90 \%(900 \mathrm{~km})$.

Tabela 6: Resultados para Linhas com Compensação em Derivação $(900 \mathrm{~km})$.

\begin{tabular}{|c|c|c|c|c|c|c|c|}
\hline \multirow{2}{*}{ Sistema } & V & $\mathbf{L}$ & R.Der & $\mathbf{r}_{0}$ & Xn & Isec & TPA \\
\hline & $(\mathrm{kV})$ & $(\mathbf{k m})$ & $(\%)$ & $\left(\frac{X_{0}}{X d}\right)$ & $(\Omega)$ & (A) & $(\mathrm{kV})$ \\
\hline \multirow{6}{*}{ 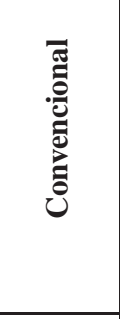 } & 500 & 900 & 80 & 2,2 & 159,32 & 57,5 & 39,8 \\
\hline & 500 & 900 & 90 & 1,9 & 106,21 & 33,3 & 26,5 \\
\hline & 500 & 900 & 95 & 1,8 & 89,44 & 29,7 & 26,2 \\
\hline & 765 & 900 & 80 & 1,0 & - & 119 & 78 \\
\hline & 765 & 900 & 90 & 1,1 & 15,22 & 100 & 74,4 \\
\hline & 765 & 900 & 95 & 1,1 & 14,41 & 94,0 & 73,4 \\
\hline \multirow{3}{*}{ 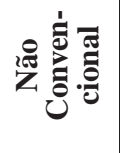 } & 500 & 900 & 80 & 1,4 & 44,99 & 104,7 & 72,7 \\
\hline & 500 & 900 & 90 & 1,4 & 39,99 & 80 & 55,2 \\
\hline & 500 & 900 & 95 & 1,4 & 37,88 & 71 & 53,2 \\
\hline
\end{tabular}

\subsection{Análise de Resultados em Linhas com Compensação Reativa em Deri- vação e Compensação Capacitiva em Série}

A figura 15 apresenta o diagrama unifilar dos sistemas com compensação reativa em derivação (banco de reator - R.Der) e compensando $50 \%$ da reatância longitudinal através de um capacitor em série (C.Sér) instalado no meio da linha.

Na tabela 7 são mostrados os valores obtidos da Isec, TPA e reator de neutro $\left(\mathrm{X}_{n}\right)$ para cada nível de compensação em derivação simulado e compensando a metade da reatância 


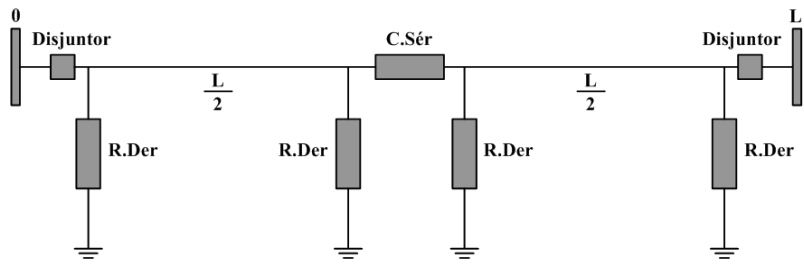

Figura 15: Diagrama Unifilar de uma Linha com Compensação Reativa em Derivação e Compensação Série no Meio da Linha.

longitudinal, para cada classe de tensão pré-estabelecida em linhas de $900 \mathrm{~km}$.

Tabela 7: Resultados para Linhas com Compensação em Derivação $(900 \mathrm{~km})$.

\begin{tabular}{|c|c|c|c|c|c|c|c|}
\hline \multirow{2}{*}{ Sistema } & V & $\mathbf{L}$ & R.Der & $\mathbf{r}_{0}$ & $\mathbf{X n}$ & Isec & TPA \\
\hline & $(\mathrm{kV})$ & $(\mathrm{km})$ & $(\%)$ & $\left(\frac{X_{0}}{X d}\right)$ & $(\Omega)$ & (A) & $(\mathrm{kV})$ \\
\hline \multirow{6}{*}{ 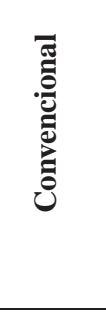 } & 500 & 80 & 50 & 2,8 & 524,28 & 61,22 & 39,24 \\
\hline & 500 & 85 & 50 & 2,6 & 438,61 & 27,6 & 23,9 \\
\hline & 500 & 90 & 50 & 2,4 & 362,46 & 12,4 & 14,5 \\
\hline & 765 & 80 & 50 & 1,4 & 151,06 & 40,0 & 31,4 \\
\hline & 765 & 85 & 50 & 1,4 & 142,17 & 27,5 & 20,5 \\
\hline & 765 & 90 & 50 & 1,4 & 134,27 & 24,8 & 20,0 \\
\hline \multirow{3}{*}{ 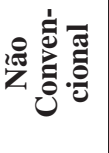 } & 500 & 80 & 50 & 2,1 & 271,77 & 74,5 & 44,33 \\
\hline & 500 & 85 & 50 & 2,0 & 232,53 & 38,8 & 30,4 \\
\hline & 500 & 90 & 50 & 1,9 & 197,7 & 21,3 & 21,4 \\
\hline
\end{tabular}

Na figura 16 mostra-se um exemplo de obtenção do valor de $\mathrm{r}_{0}$ ótimo $(2,6)$ para o sistema de $500 \mathrm{kV}$ convencional com comprimento $900 \mathrm{~km}, 50 \%$ de compensação série e grau de compensação reativa $85 \%$. Este valor ótimo de $\mathrm{r}_{0}$ foi obtido encontrando a Isec máxima para faltas ao longo da linha ao variar-se $r_{0}$. Na figura 17 apresentam-se Isec e TPA para faltas ao longo da linha como $\mathrm{r}_{0}$ ótimo.

Com a nova proposta de compensação reativa das linhas longas, compensando o reativo longitudinal e distribuindo a compensação reativa transversal, foi possível encontrar um valor ótimo de reator de neutro para cada sistema de transmissão que reduzisse os valores da Isec a valores muito baixos, assegurando uma alta probabilidade de auto-extinção do arco.

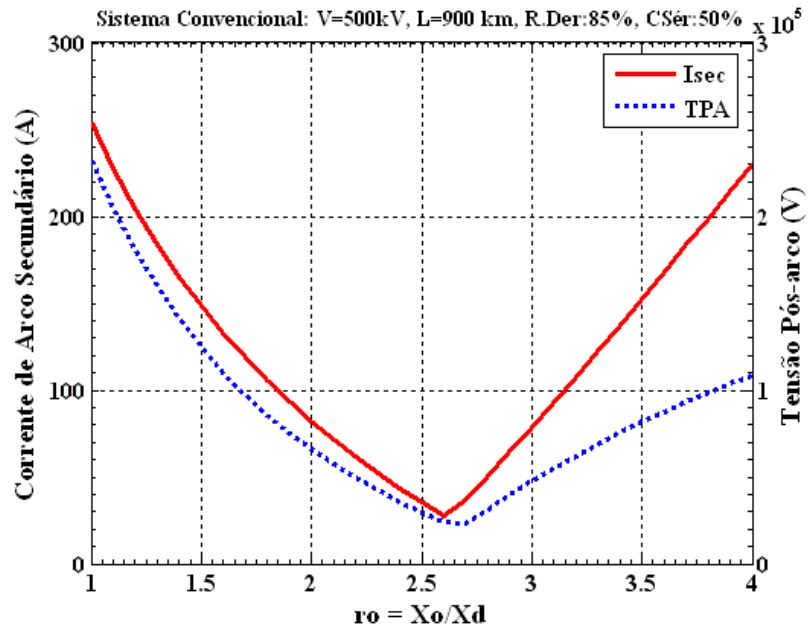

Figura 16: Valores Máximos de Isec e TPA em função do "ro" - Linha 500 kV Convencional com Compensação em Derivação: 85 \% e Compensação Série: $50 \%$ (900km).

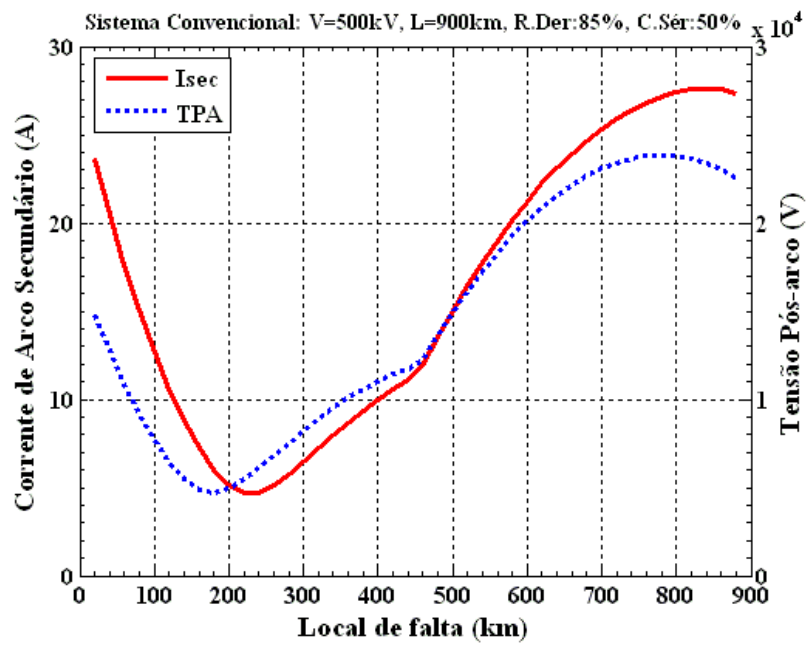

Figura 17: Isec e TPA ao Longo da Linha para o Reator de Neutro Ótimo $\left(\mathrm{r}_{0}=2,6\right)$ - Linha $500 \mathrm{kV}$ Convencional com Compensação em Derivação: 85 \% e Compensação Série: $50 \%(900 \mathrm{~km})$.

\section{CONCLUSÕES}

No presente trabalho realizamos uma extensa análise de sensibilidade da corrente de arco secundário que surge durante a manobra de abertura monopolar para eliminação de falta monofásica não permanente. Diversos sistemas de transmissão compostos por linha mais sistema de compensação foram avaliados, sendo os principais resultados resumidos a seguir:

- Para as linhas curtas estudadas, de $150 \mathrm{~km}$, para as quais não há necessidade de efetuar compensação re- 
ativa, existe uma elevada probabilidade do arco secundário se extinguir durante a manobra monopolar devido ao baixo valor da corrente de arco secundário.

- Para as linhas com $450 \mathrm{~km}$ foi necessário introduzir a compensação reativa para a redução da tensão da linha em vazio. Para estes sistemas o correto dimensionamento do reator de neutro foi suficiente para reduzir a corrente de arco secundário sustentada para níveis adequados para permitir a manobra monopolar com segurança.

- Para linhas longas, com $900 \mathrm{~km}$ foi verificado que:

- Para o sistema convencional de 500 kV foi possível dimensionar o reator de neutro da compensação reativa localizada nos extremos da linha para reduzir a corrente de arco secundário sustentada a níveis adequados aos critérios estabelecidos. No entanto o nível de compensação analisado foi elevado.

- Para o sistema não convencional de 500 kV e o sistema convencional de $765 \mathrm{kV}$ foi necessário compensar o reativo longitudinal e dividir a compensação reativa transversal em 04 pontos ao longo da linha. Para o novo arranjo da compensação o adequado dimensionamento do reator de neutro permitiu reduzir a corrente de arco secundário sustentada a valores que indicam uma elevada probabilidade de sucesso na manobra monopolar.

Para cada sistema de transmissão deve-se realizar um estudo específico para verificar a viabilidade de se efetuar a manobra monopolar. Não se pode generalizar um mesmo intervalo de relação " $\mathrm{r}_{0}$ " para sistemas com a mesma classe de tensão ou linhas com o mesmo comprimento.

\section{AGRADECIMENTOS}

Os autores agradecem o apoio financeiro dos órgãos de pesquisa: Coordenação e Aperfeiçoamento de Pessoal de Nível Superior (CAPES), Conselho Nacional de Desenvolvimento Científico e Tecnológico (CNPq) e à Fundação de Amparo à Pesquisa do Estado de São Paulo (FAPESP).

\section{REFERÊNCIAS}

EPRI (1982). Transmission Line Reference Book $345 \mathrm{kV}$ and Above. $2^{\text {nd }}$ ed, Palo Alto - California.

Gonzales, A.J., C. Kung, C. Raczkowski, C.W. Tylor and D. Thonn (1984). Effects of Single and three Pole Switching and High Speed reclosing on Turbine Generator
Shafts and Blades. IEEE Trans Power Apparatus and Systems, Vol. PAS-103, No. 11, pp. 3218-3228.

Kimbark, E.W. (1964). Suppressions of Ground-Fault Arcs on Single-Pole-Switched EHV Lines by Shunt Reactors. IEEE Transactions Power Apparatus and Systems, Vol.83, No. 2, pp. 285-290.

Kimbark, E.W. (1976). Selective-pole Switching of Long Double-circuit EHV Line. IEEE Transactions Power Apparatus and Systems, Vol.PAS-95, No. 2, pp. 219230.

Portela, C.M. (1980). A Study of Problems Related to Switching of Relatively Small Currents. CIGRE PTEL 1305, Anexo 6.2-2003, Brasil.

Portela, C.M., H.C. Santiago, O.B. Oliveira and C.J. Dupont (1992). Modeling of Arc Extinction in Air Insulation. IEEE Transactions On Electrical Insulation. Vol.-27, No. 3, pp. 457-463.

Tavares, M.C. and C.M. Portela (2004). Transmission System Parameters Optimization-Sensitivity Analysis of Secondary Arc Current and Recovery Voltage. IEEE Transactions on Power Delivery, Vol.19, No. 3, pp. 1464-1471.

Zevallos, M.E. (2007). Análise de Sensibilidade da Corrente de Arco Secundário para Diferentes Linhas de Transmissão. Dissertação de Mestrado, Departamento de Sistemas de Controle e Energia, DSCE-FEEC-UNICAMP, Campinas - SP. 